\title{
REUSO DE AGUA NA INDÚSTRIA DE PETRÓLEO E GÁS
}

\author{
S. BORSCHIVER ${ }^{1}$, A.O.C. JESUS ${ }^{2}$ e V.A. MOREIRA ${ }^{3}$ \\ Universidade Federal do Rio de Janeiro, Escola de Química, Núcleo de Estudos \\ Industriais e Tecnológicos (NEITEC) \\ E-mail para contato: suzana@eq.ufrj.br ${ }^{1}$, andressaocj@ufrj.br ${ }^{2}$, \\ moreira.a.viviane@gmail.com ${ }^{3}$
}

$\mathrm{O}$ reuso de água vem ganhando um grande destaque na indústria química. Os tratamentos de águas residuais de refinarias podem ser classificados como primários secundários e terciários. Métodos primários consistem em separações físicas; os secundários, na remoção de grande parte de material biodegradável e de sólidos biológicos e os terciários visam à remoção de íons. O trabalho apresenta os cenários de desenvolvimento tecnológico no reuso de água na indústria de óleo e gás, com ênfase no setor de up stream (refinaria) mediante a análise de documentos técnicos, como artigos científicos e patentes. A ferramenta de informação utilizada para artigos foi o Scirus e, para as patentes concedidas e solicitadas, o USPTO, de 2008 a 2013. Irã, Brasil e China foram os países com o maior número de artigos publicados, sendo que o tema mais frequente foi o tratamento terciário, com $56 \%$ do total. Cerca de $80 \%$ das patentes concedidas e solicitadas originam-se dos EUA, com destaque para as empresas EXXON MOBIL, TEXACO, NALCO e GE, também em tratamento terciário. Nas tecnologias de membrana observou-se tanto processos de separação quanto de conversão, com destaque para as empresas Biological Petroleum Clean, de Israel e Aptwater dos EUA.

\section{INTRODUÇÃO}

Existe atualmente um cenário de estresse sobre os recursos hídricos, causado pelo intenso crescimento populacional, acompanhado de um aumento da demanda de água em diversas indústrias. A indústria do petróleo e gás natural, e, em particular, o setor de refino e petroquímica, demandam uma quantidade significativa de água para o exercício de suas atividades. Em média, o volume de água utilizado para o processamento de um barril de petróleo em uma refinaria varia de 250 a 300 litros. (Szklo, 2005).

No passado, o principal objetivo e preocupação da produção de óleo eram a exploração e produção de petróleo e de gás, mas, ao longo dos anos, a preocupação com a produção de água também surgiu, influenciada principalmente pelas pressões ambientais. Assim, os estudos para obter as melhores tecnologias para o tratamento da água estão em 
evidência visando tanto uma maior eficiência na remoção e recuperação de petróleo e outros agentes tóxicos, bem como na viabilidade financeira destas tecnologias.

As plantas de refino de petróleo são grandes consumidoras de água. No Brasil, durante o ano de 2009, foram consumidos 254.093 de $\mathrm{m}^{3} /$ dia de água, que são usados em sistemas de geração de vapor, torres de refrigeração, combate a incêndios e fins de consumo. Assim, o reúso de água surge como uma alternativa interessante, tanto no sentido de minimizar sua utilização como no de reduzir a carga poluente. Além disso, com a implementação dos mecanismos de outorga e cobrança pela utilização dos recursos hídricos no Brasil (Política Nacional de Recursos Hídricos - PNRH, Lei N ${ }^{\circ}$ 9.433, de 8 de janeiro de 1997), o reúso de efluentes nas indústrias passou a ser sinônimo de ganhos econômicos diretos, além de ser responsável por uma melhoria na imagem das empresas. Outra legislação importante consiste na Resolução CONAMA $\mathrm{N}^{\mathrm{o}} 357$, de 17 de março de 2005, que estabelece as condições e padrões de lançamento de efluentes. (Pombo,2011).

$\mathrm{O}$ reuso de água pode ser realizado das seguintes maneiras: o efluente pode ser utilizado diretamente em outras operações, quando o nível de contaminação não interfere no próximo processo, ou na regeneração, reutilização do efluente tratado em outro processo.

Como as plantas de refino de petróleo são grandes consumidoras de água, elas necessitam de estudos para garantir o desenvolvimento de medidas para racionalizar o uso da mesma. A água é utilizada em refinarias de petróleo em todo o mundo, em duas aplicações principais: torres de resfriamento e caldeiras (geração de vapor), correspondendo a $60-70 \%$ e $20-30 \%$, respectivamente. Os outros usos da água são as unidades de fins do processo, combate a incêndios e de consumo, correspondem a $9 \%$. As principais unidades de consumo de água das refinarias de petróleo são: Dessalinização, destilação do óleo cru, craqueamento termal, craqueamento catílico, hidrocraqueamento, polimerização, isomerização, reforma e hidrotratamento.

\section{OBJETIVO}

O objetivo do presente trabalho é realizar um estudo de prospecção tecnológica em Reuso de água para a indústria de petróleo e gás, mais especificamente, no setor de up stream, de modo a delinear o cenário tecnológico dos principais players envolvidos, as tecnologias maduras, emergentes, estudos de bancada, planta piloto e os principais insumos dos processos do reuso.

\section{METODOLOGIA}

A metodologia foi baseada em técnicas de prospecção tecnológica, onde se realizou a busca e análise de artigos no Scirus, no período de 2004 a 2013, e de patentes no 
USPTO (United States Patent and Trademark Office's) no período de 1976 a 2013. As informações foram organizadas nos parâmetros Macro, Meso e Micro, onde a análise Macro abrange a série histórica das publicações, os países que têm mais artigos publicados no período, as revistas mais importantes, universidades, centros de pesquisa e empresas relacionadas ao assunto. Na análise Meso os documentos são categorizados de acordo com os aspectos mais relevantes em torno do assunto. Na análise Micro cada fatia da análise Meso é detalhada e são então identificadas particularidades. A análise Micro depende dos resultados apresentados nas análises anteriores, gerando informações sobre as tecnologias utilizadas em casa análise Meso.

\subsection{A prospecção tecnológica}

Os Estudos de Prospecção Tecnológica, também chamados de estudos de futuro, ou forecast(ing), foresight(ing) ou future studies, fornecem as principais tendências no contexto mundial sendo possível segmentar estas tecnologias por setor da economia. Estes estudos auxiliam a identificação de tecnologias promissoras, úteis para uma determinada organização, bem como apontam para possibilidades de negócios e parcerias. A sistematização da prática de monitoramento tecnológico, a ser coberta pela prospecção tecnológica e de inovação, visa congregar a busca de soluções adequadas para a identificação e priorização de uma agenda de $\mathrm{P} \& \mathrm{D}$, articulada com instituições de pesquisa, que possa inclusive influenciar agenda de $\mathrm{P} \& \mathrm{D}$ nacional e criar demandas para a cadeia inovativa do setor. (Borschiver,2008)

Os objetivos de um estudo prospectivo podem ser bem abrangentes, pois podem ir desde futuros relacionados a um universo mais amplo, onde uma grande quantidade de atores está envolvida e existe uma grande quantidade de fatores e variáveis que devem ser analisados, até um universo mais limitado, onde empresas ou organizações objetivam gerar estudos prospectivos que analisem os fatores exógenos ao ambiente daquela empresa ou organização. Com tais estudos as empresas buscam verificar o grau de influência destes fatores nos seus produtos e no seu nicho de mercado. Tais estudos devem servir de ferramenta não só para apoiar os processos de planejamento corporativo das empresas, mas também as políticas públicas e setoriais. (Borschiver,2008)

\section{RESULTADOS}

\subsection{Artigos}

A busca de artigos no SCIRUS foi baseada nas palavras "refinery wastewater" e "reuse". Foram encontrados 207 artigos e dentre esses, 66 foram pertinentes ao tema. Os principais anos de publicação foram 2008, 2011 e 2013. A maioria dos artigos 
analisados foi publicada por universidades ou centros de pesquisas. Apenas $16 \%$ desses artigos pertencem a empresas.

Dentre os países que mais tiveram publicações destacam-se o Iran, com 14 artigos, seguido da China, com 10 e Brasil, com 8. Pode ser mencionado um artigo iraniano da parceria entre Islamic Azad University e Arak University sobre a utilização de óxidos metálicos, como calamina e o dióxido de titânio, juntamente com radiação ultravioleta para tratamento terciário de efluentes da refinaria de Bandar Abbas.

Em relação à China, destaca-se um estudo da Dalian University of Technology sobre um processo de oxidação catalítica assistida por micro-ondas para tratar efluentes de refinaria, utilizando carvão ativado granulado como catalisador.

No caso do Brasil, grande parte do material encontrado foi da UFRJ em parceria com o Cenpes-Petrobrás. Um exemplo é a avaliação de um MBBR (Moving Bed Biofilm Reactor) e a possibilidade de reuso desse efluente, após ozonização em série com uma coluna de carvão ativado biológico.

Após uma análise mais aprofundada nos artigos encontrados, observou-se que os temas abordados eram: "Tecnologia" (48\%), "Equipamentos" (23\%), "Membrana" (17\%), "Automação" (7\%) e "Catalisador" (5\%).

As empresas que mais publicaram sobre "Tecnologia" foram: Petrobrás, Academic Center for Education, Culture and Research, Isfahan Water and Wastewater Company, Serionix, UNICEF em parceria com Pakistan Environmental Protection Agency, National Iranian Oil Refining and Distribution Company e Liaohe Petrochemical Branch Company.

As tecnologias encontradas podem ser divididas entre tratamento terciário, encontrado em 55\% das publicações, tratamento secundário, presente em $32 \%$ e tratamento primário, em $13 \%$ dos documentos analisados.

Em relação às empresas que publicaram sobre "Equipamentos", encontram-se: Luoyang Petrochemical Engineering Corporation, National Iranian Oil Refining and Distribution Company, Liaohe Petrochemical Branch Company, e Petrobrás. Pode-se destacar um estudo da China (uma parceria entre Luoyang Petrochemical Engineering Corporation e Tianjin University) que refere-se a equipamento e membrana, com um tratamento aeróbico realizado em um biorreator em escala piloto (Airlift Loop Bioreactor - ALR) com fluxo em três fases (sólido, líquido e gasoso) em que a membrana biológica substitui as lamas ativadas.

Dentre os equipamentos mais utilizados, estão SBR - Sequencing Batch Reactor, biorreatores (não especificados) e eletrodos. Alguns dos estudos de SBR referem-se à automação também, como, por exemplo, um estudo de comparação entre diferentes tipos de controle de oxigênio dissolvido em uma planta piloto de SBR. 
A respeito de "Membrana" as empresas que publicaram foram: Petrobras, Luoyang Petrochemical Engineering Corporation, Academic Center for Education, Culture \& Research e Servizi Porto Marghera Scarl. Pode ser mencionado um estudo da PetrobrasCenpes sobre um tratamento de um efluente da refinaria utilizando um biorreator de membrana submersa (SMBR).

Os processos de tratamento com membranas foram divididos entre conversão e separação. Os processos de conversão referem-se aos reatores utilizando membranas e podem ser mencionados biorreatores à membrana, biorreatores à membrana anaeróbicos e reator à membrana em processo de batelada (Membrane Sequencing Batch Reactor MSBR). Já os processos de separação podem ser divididos entre ultrafiltração, microfiltração, osmose inversa e nanofiltração.

Um dos estudos sobre ultrafiltração é do Instituto Tecnológico Indiano e tem o foco principal no tratamento do efluente por membranas de ultrafiltração polissulfonadas modificadas por aditivos como polietilenoglicol.

\subsection{Patentes concedidas}

A busca de patentes foi realizada no USPTO utilizando as seguintes palavras-chave: treatment e reuse, combinadas com wastewater. Foram analisadas 177 patentes e 60 apresentaram foco no tema.

Os EUA foram responsáveis por $85 \%$ das patentes publicadas. Houve um maior número de publicações nos anos 1996,2000 e 2012.

As empresas foram responsáveis pela maioria das patentes, com destaque para Exxon Mobil, com 16\% das patentes e para Texaco e Nalco Chemical Company, cada uma com $11 \%$, todas essas com atuação na indústria de óleo e gás.

A figura a seguir mostra que $69 \%$ das patentes consideradas no estudo tem foco em "Tecnologia". As patentes com foco em "Membrana" representam 13\%, enquanto "Equipamento" representam 12\%. Patentes como foco em "Automação" e "Catálise" representam $5 \%$ e $1 \%$ respectivamente. 


\section{FOCO DO DOCUMENTO}

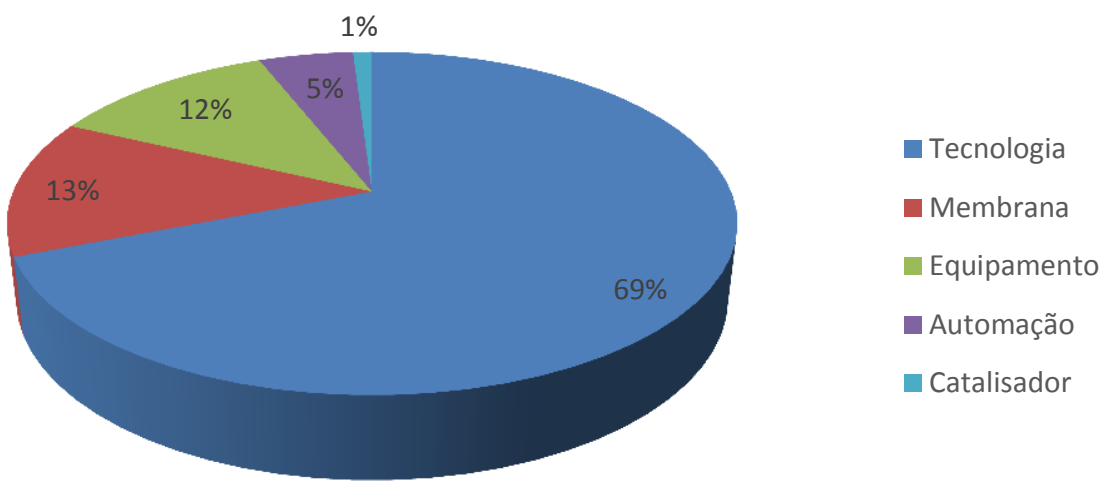

Figura 1- Foco dos documento

Pode-se destacar 3 empresas com patentes com foco em "Tecnologia", são elas: Exxon Mobil com 9 patentes e Texaco e Nalco com 6 patentes cada. Em relação a "Membrana", pode-se destacar a APT Water, Texaco e Biological Petroleum Cleaning, cada uma com 2 patentes com foco no assunto. "Equipamento" foi abordado por 7 diferentes empresas, British Petroleum Company, Tidwell Construction Company, Combustion Engineering, The Standard Oil Company, International Environmental Systems, Texaco e Hydrotreat. "Automação" foi citada em 4 patentes, onde 2 delas também falavam de "Membrana" e foram publicadas pela Biological Petroleum Cleaning e as outras 2 também citavam "Equipamento" e foram publicadas pela Tidwell Construction Company e Texaco. Somente uma patente abordou "Catalise" e essa foi publicada pela Exxon Mobil.

O tratamento primário é mais frequente, representando cerca de $42 \%$ do total, enquanto o terciário representou $41 \%$ e o secundário apenas $17 \%$.

Patentes que abordam "Membrana" apresentam, assim como nos artigos, processo de separação ou processo de conversão. $64 \%$ das patentes encontradas abordam processos de separação, enquanto $36 \%$ processos de conversão. Os processos de separação por membrana encontrados na pesquisa foram: membrana por osmose reversa, membrana de ultrafiltração, processo com membrana de fibra oca, filtro de membrana, microfiltração por membrana e membrana resiliente. Os processos de conversão foram tratados em apenas 2 patentes, uma em relação a biorreator de membrana e outra com Reator de membrana biofilme.

Dentre os equipamentos utilizados no processo de reuso de água, pode-se destacar: separador, CSTR, vaso de pressão, biorreator e contactor vessel. Em relação à "Automação", foram encontradas apenas três patentes, tendo como principais atuadores do processo válvulas controláveis, controlador de $\mathrm{PH}$, controlador de razão de fluxo, sensores, além de um controlador eletrônico. 
Com foco no "Catalisador", houve apenas uma patente, de 1996, da Mobil Oil Corporation, sobre oxidação catalítica que envolve contato de águas residuais com uma fonte de oxigênio sobre um catalisador (NiMo, NIW e Como) composta por uma combinação de um componente do Grupo VIII e um metal Grupo VIA, em um suporte que seja inerte nas condições hidrotérmicas do tratamento de águas residuais.

\subsection{Patentes Solicitadas}

Em relação as patentes solicitadas também utilizou-se como palavras-chave combinações de Refinery water, Refinery wastewater, Membrane, Treatment, Handling, processing, removal e reuse. Foram analisadas 186 patentes e 34 apresentaram foco no assunto desejado.

Os EUA é o país com maior número de patentes solicitadas (75\%), seguido do Japão (6\%); e França e Índia, (5\%). Entre as empresas, as que tiveram maior número de publicações foram a GE com 8\%, ExxonMobil e ConocoPhilips com 6\% das patentes cada. $\mathrm{O}$ restante foi solicitado por pessoa física. Assim com nas patentes concedidas, foi observado que as empresas que mais solicitaram patentes sobre o assunto atuam no setor de óleo e gás.

Em relação aos assuntos principais dos documentos, o resultado foi semelhante, percentualmente, em comparação com as patentes concedidas, como mostra o gráfico a seguir. "Tecnologia" estava no topo (31 patentes, dos quais 18 foram solicitadas por empresas), e a maioria tratamento terciário, seguido por "Membrana" (13, dos quais 6 foram solicitadas por empresas), "Equipamento" (10, dos quais 5 foram solicitadas por empresas) e "Automação" (2 com 1 solicitada por empresa).

\section{FOCO DO DOCUMENTO}
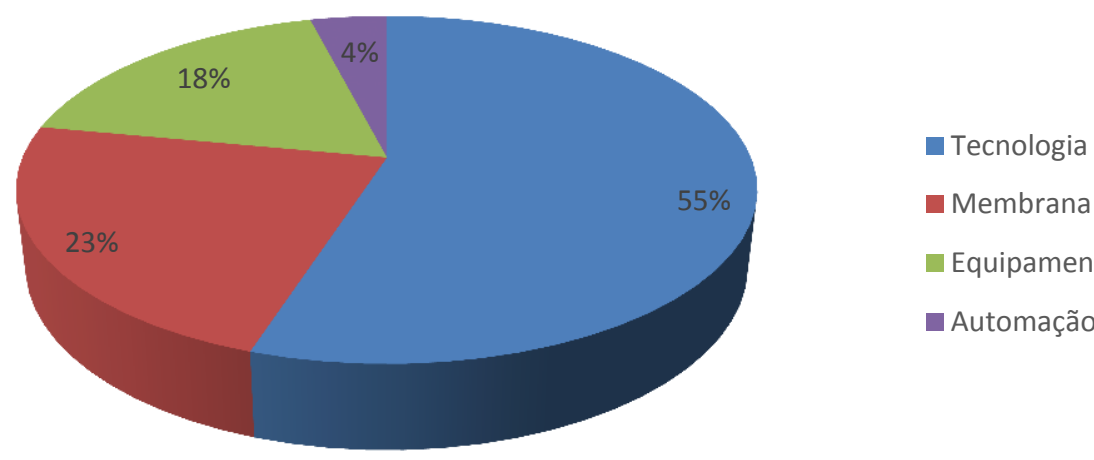

- Membrana

Equipamento

- Automação

Figura 2- Foco dos documentos. 
A maior parte dos processos de "Membrana" são processos de separação (79\%), com destaque para membrana de osmose reversa e filtro de membrana. Os equipamentos identificados na análise das patentes solicitadas foram: tanque de mistura, separador, subsistema de filtração, biorreator, reator fotocatalítico e dispositivo de separação por gravidade. Sobre "Automação", foram encontradas apenas duas patentes, tendo como principais atuadores do processo controladores e dispositivos automáticos e programados, com destaque para a empresa Biological Petroleum Cleaning.

\section{CONCLUSÃO}

Após a análise do trabalho, foi observada uma grande tendência de desenvolvimento no setor de reuso de água na indústria de petróleo e gás nos EUA, visto que foi o país que publicou a grande maioria de patetes, solicitadas e concedidas, o que representa indicadores de desenvolvimento a curto e médio prazo. A maioria das patentes presentes na pesquisa, são de empresas que atuam no setor de óleo e gás como Exxon Mobil, GE e Texaco. Os artigos, que são indicadores de métodos utilizados atualmente ou tendências de desenvolvimento a longo prazo, foram publicados em sua maioria, por universidades e/ou centros de pesquisas, de países como Iran, China e Brasil. O estudo de "Tecnologia" é o foco de mais da metade dos documentos analisados, com destaque para as tecnologias primárias e terciárias. Os estudos de "Membrana" e "Equipamento" também apresentam grande quantidade de documentos, enquanto a minoria tem como foco "Automação" e "Catalisador". Estudos como esse corroboram com o fato da importância do tema em questão bem como da metodologia de prospecção tecnológica aplicada.

\section{REFERÊNCIAS}

Borschiver, S. Apostila de Curso de Pós Graduação, EQ/UFRJ, 2008

Pombo, F. R. Water Demand Management in Petroleum Refining Industry: Challenges and Opportunities for Rationalization, Federal University of Rio de Janeiro, Brazil, 2011

Szklo, Alexandre Salem. Fundamentos do Refino de Petróleo. Editora Interciência. Rio de Janeiro. 2005. 